\title{
Application of the international league against rheumatism classification criteria for systemic juvenile idiopathic arthritis as a prognostic factor in patients with adults-onset Still's disease
}

\author{
Ji Won Yang ${ }^{1}$, Eunyoung Lee ${ }^{2}$, Ji-Yeon Seo ${ }^{1}$, Ju-Yang Jung ${ }^{1}$, Chang-Hee Suh ${ }^{1}$ and Hyoun-Ah Kim ${ }^{1 *}$ [D
}

\begin{abstract}
Background: Adult-onset Still's disease (AOSD) is an adult form of systemic juvenile idiopathic arthritis (JIA) that differs from the latter in its classification. This study evaluated the concordance between the International League Against Rheumatism (ILAR) criteria for systemic JIA and the Yamaguchi criteria and then compared their possible prognostic value in patients with AOSD.

Methods: In a retrospective review of 169 adults with suspected AOSD, patients were classified according to the Yamaguchi or ILAR criteria. Then the concordance in cross-referencing the other group with the different criteria was investigated and the sensitivity and specificity of each set of criteria were determined. Disease activity markers in AOSD patients were correlated with positivity according to both systems.

Results: Concordance was good in patients with suspected AOSD ( $k=0.7144, p<0.001)$ and low in those with a diagnosis of AOSD $(k=0.3787, p<0.001)$. The sensitivity of the ILAR criteria in AOSD patients was 0.8864 (95\% confidence interval (Cl): 0.8322-0.9405), and the specificity was $0.7838(0.6511,0.9164)$. Positivity according to the ILAR criteria correlated with the systemic score $(r=0.763, p<0.0001)$ and C-reactive protein levels ( $r=0.183, p=0.0356)$ and was associated with a relapse (odds ratio: $1.589,95 \% \mathrm{Cl}: 1.043-2.421$ ), macrophage activation syndrome (MAS; odds ratio: 1.993, 95\% Cl: 1.218-3.263) and care in the intensive care unit (ICU; odds ratio: 2.087, 95\% Cl: 1.086-4.011).

Conclusions: In AOSD patients, there is fair concordance between the Yamaguchi and ILAR criteria for systemic JIA. Positive ILAR criteria may be useful for identifying AOSD patients at high risk for relapse, MAS and the need for ICU care. Further studies including larger populations from several centers are needed to confirm our results regarding the utility of the ILAR criteria in AOSD patients.
\end{abstract}

Keywords: Adult-onset Still's disease, Systemic juvenile idiopathic arthritis, Yamaguchi criteria, ILAR criteria

\footnotetext{
*Correspondence: nakhada@naver.com

'Department of Rheumatology, Ajou University School of Medicine, 164

Worldcup-ro, Yeongtong-gu, Suwon 16499, South Korea

Full list of author information is available at the end of the article
} 


\section{Background}

Still's disease is a systemic form of juvenile idiopathic arthritis (JIA), the etiology and pathogenesis of which are unknown. Systemic JIA is a cause of fever of unknown origin (FUO) and it is accompanied by several systemic manifestations, such as arthritis, an evanescent rash, and serositis. A cohort of 14 adults who presented with the same symptoms as JIA patients was described in 1971, and the name adult-onset Still's disease (AOSD) was proposed [1]. Although the adult and pediatric diseases are considered parts of the same spectrum, the diagnosis of one or the other depends on the age at onset. Individuals presenting at the age of 16 years and older are diagnosed with AOSD; younger individuals are diagnosed with systemic JIA [2,3]. However, there are differences between JIA and AOSD, such as a higher seasonality in the former and a higher rate of pharyngitis in the latter [4]. A recent study compared the cytokine profiles of patients with AOSD and systemic JIA [5]. Among the shared features was a significant increase in interleukin 18 (IL-18) levels. Differences between the IL6- and IL-18-based cytokine profiles may be responsible for the different clinical manifestations of JIA and AOSD and suggest the presence of two distinct subgroups within a single disease category [6].

AOSD is often difficult to diagnose because patients may present with several nonspecific symptoms and there are no serologic biomarkers of the disease. While there are several sets of clinical criteria for the classification of AOSD, those of Yamaguchi are used most widely and consist of fever, arthralgia, typical rash, and leukocytosis as major criteria, and sore throat, lymphadenopathy or splenomegaly, liver dysfunction, and the absence of rheumatoid factor (RF) and antinuclear antibody (ANA) as minor criteria [7]. Some manifestations are related to an unfavorable outcome after a chronic disease course and a prolonged period of time until clinical remission, such as typical rash, root joint arthritis, and polyarthritis [8].

AOSD and systemic JIA are considered similar diseases with a shared pathogenesis and gene expression profile, but they are difficult to classify according to the criteria currently available $[5,7,9]$. Among the various clinical criteria for diagnosing AOSD, the Yamaguchi criteria are commonly used in clinical practice, and are the most widely cited; however, they require the prior exclusion of neoplasms, infections, and autoimmune diseases mimicking AOSD $[7,10,11]$. The International League Against Rheumatism (ILAR) first proposed the term JIA, which includes all forms of arthritis that onset before the age of 16 years, persist for more than 6 weeks, and are of unknown origin [12, 13]. Although the ILAR classification of JIA is widely used, it has the limitation of being an incomplete system and new criteria have been proposed for the classification of JIA, including systemic onset $[14,15]$. One study evaluated the efficacy of substituting adult diagnostic criteria in the evaluation of pediatric patients with suspected systemic JIA [16]. The results suggested that the Yamaguchi criteria can be useful for the subset of patients in the pre-arthritic phase of the disease. However, few studies have examined the utility of the ILAR criteria for the classification of patients with AOSD. Thus, in this retrospective study, we assessed the compatibility of the ILAR criteria for systemic JIA when applied to patients with AOSD. Conversely, we asked whether the ILAR criteria could be used to diagnose, evaluate disease activity, and predict the prognosis in patients with AOSD.

\section{Methods \\ Patients}

We identified 191 patients within the Ajou University Hospital computer system with the diagnostic code for AOSD; 22 of these patients were excluded from this study because of missing or insufficient data. We retrospectively reviewed 169 patients with suspected AOSD at the time of the initial visit to Ajou University Hospital between 2001 and 2017. During the diagnostic evaluation, 37 patients were diagnosed with another disease, including viral infection, palindromic rheumatism, lupus-like disease, and Kikuchi's disease. Inclusion in the diagnosis of AOSD required (1) a diagnosis code of AOSD retained by the referring physician at the last follow-up; (2) a diagnosis of AOSD retained by the investigator (H.-A. K.); and (3) meeting none of the classification criteria for other immune-mediated inflammatory diseases previously described [10]. The 132 remaining patients with a diagnosis of AOSD were compared with the 37 patients with other diagnoses. All enrolled patients with five or more of the Yamaguchi criteria (and fulfilling at least two major criteria) were classified after excluding infections, malignancies (especially malignant lymphoma), and other rheumatic diseases [7]. The major and minor Yamaguchi criteria are as follows. The major criteria are (1) fever of at least $39^{\circ} \mathrm{C}$ for at least 1 week, (2) arthralgia or arthritis for at least 2 weeks, (3) non-pruritic salmon-colored rash on the trunk/ extremities, and (4) granulocytic leukocytosis $(10,000 / \mathrm{mL}$ or greater). The minor criteria are (1) sore throat, (2) lymphadenopathy, (3) hepatomegaly or splenomegaly, (4) abnormal liver function tests, and (5) negative in tests for RF and ANA. Then, these patients were reclassified using the ILAR criteria for systemic JIA [12] and the concordance between the ILAR and Yamaguchi criteria was assessed. A daily fever exceeding $39{ }^{\circ} \mathrm{C}$ for at least 2 weeks or arthritis in one or more joints lasting for at least 6 weeks accompanied by one or more of the following: (1) evanescent erythematous rash, (2) generalized lymph node enlargement, (3) hepatomegaly or splenomegaly, and (4) serositis. We also evaluated the sensitivity and specificity of the ILAR criteria. This study was 
approved by the Institutional Review Board of our hospital (AJIRB-MED-MED-17-297).

\section{Variables}

All clinical data were retrieved from patient medical records stored in the hospital's database. Clinical characteristics, including age, sex, clinical symptoms, follow-up period, organ involvement and extent, treatment, and outcome were evaluated. Each patient underwent a series of laboratory tests, including a complete blood count $(\mathrm{CBC})$ and determination of the erythrocyte sedimentation rate (ESR) and levels of Creactive protein (CRP), RF, ANA, ferritin, albumin, lactate dehydrogenase (LDH), and complement factors. AOSD disease activity was evaluated using the method of Pouchot et al., which assigns a score from 0 to 12 and adds 1 point for each of the following manifestations: fever, typical rash, pleuritis, pneumonia, pericarditis, hepatomegaly or abnormal liver function tests, splenomegaly, lymphadenopathy, leukocytosis $\geq 15,000 / \mathrm{mm}^{2}$, sore throat, myalgia, and abdominal pain [8]. Patients were treated in four steps: 1) patients used only low-dose corticosteroids or nonsteroidal antiinflammatory drugs (NSAIDs); 2) high-dose corticosteroids without disease-modifying antirheumatic drugs (DMARDs); 3) DMARDs used regardless of the use of corticosteroids; and 4) patients were treated with corticosteroids, as well as tumor necrosis factor (TNF) inhibitors, IL-6 blockade or intravenous immunoglobulin (IVIG), regardless of the use of DMARDs (Fig. 1).

\section{Statistical analysis}

The data are expressed as the mean \pm standard deviation (SD). A $P$ value $<0.05$ was considered to indicate statistical significance. The sensitivity and specificity of the two sets of diagnostic criteria were tested in patients diagnosed with AOSD group and in the non-AOSD group. The agreement between the Yamaguchi and ILAR criteria was assessed according to the kappa ( $\mathrm{k}$ ) value: a $\mathrm{k}$-value $>0.8$ indicated excellent agreement, $0.61-0.8$ meant good agreement, $0.21-0.4$ indicated fair agreement, and $<0.2$ was considered poor agreement [17]. A multivariate analysis of the significant markers was performed to determine those that were independently associated with the prognosis of AOSD. A Pearson correlation test was used to determine the correlation between ESR, CRP, ferritin, and leukocyte counts, as disease activity markers, and the Yamaguchi and ILAR criteria. All statistical analyses were two-sided and were carried out using SAS statistical software, ver. 9.4 (SAS Institute).

\section{Results}

Characteristics of the study population

Table 1 shows the clinical characteristics of the 169 patients seen at the hospital for suspected AOSD. Within this group, 132 patients were diagnosed with AOSD and 37 were diagnosed with another disease. In total, 12 patients were diagnosed with palindromic rheumatism, 11 with lupus-like disease, 4 with viral exanthema, 2 each with drug eruptions, Kikuchi's disease, and systemic lupus erythematosus, and 1 each with breast cancer, thyroid cancer, human immunodeficiency virus infection, and hypereosinophilic syndrome. The main clinical features of the 169 patients were fever (98\%), maculopapular skin rash (76\%), arthralgia (89\%),

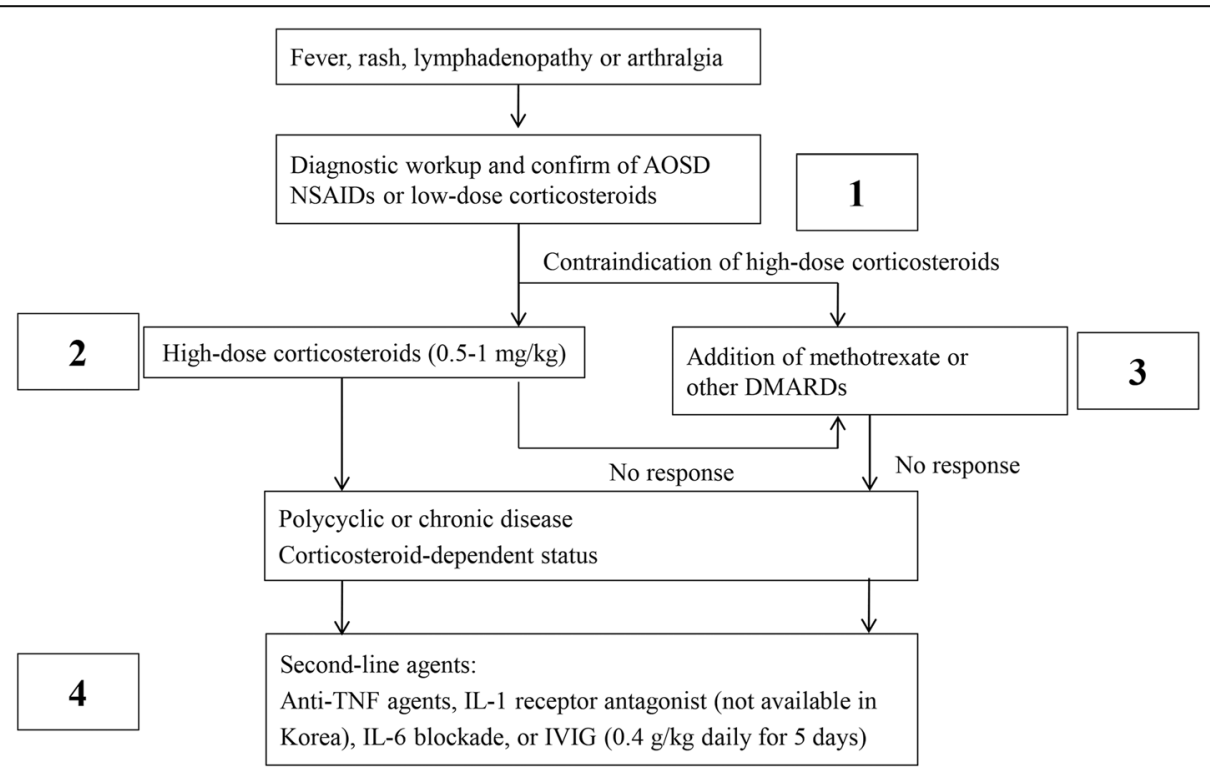

Fig. 1 A diagram for 4-step treatment schedule in patients with adult-onset Still's disease (AOSD). IVIG, intravenous immunoglobulin; IL-1, interleukin-1 
Table 1 Demographic and baseline characteristics of patients with suspected adult-onset Still's disease (AOSD)

\begin{tabular}{|c|c|c|c|}
\hline Characteristics & $\begin{array}{l}\text { AOSD suspected, } \\
\text { but not diagnosed }(N=37)\end{array}$ & AOSD patients $(N=132)$ & $P$-value \\
\hline Age (year) & $39.4 \pm 12.8$ & $41.9 \pm 15.9$ & 0.37 \\
\hline Sex (Male) & $15(41 \%)$ & $38(29 \%)$ & 0.17 \\
\hline Fever & $29(78 \%)$ & 130 (98\%) & $<.001$ \\
\hline Sore throat & $11(30 \%)$ & $71(54 \%)$ & 0.010 \\
\hline Maculopopular skin rash & $12(32 \%)$ & $102(77 \%)$ & $<.001$ \\
\hline Lymphadenopathy & $5(14 \%)$ & $45(34 \%)$ & 0.015 \\
\hline Splenomegaly & $4(11 \%)$ & $29(22 \%)$ & 0.130 \\
\hline Hepatomegaly & $2(5 \%)$ & $17(13 \%)$ & 0.200 \\
\hline Pericarditis & $0(0 \%)$ & $11(8 \%)$ & 0.069 \\
\hline Pleuritis & $0(0 \%)$ & $11(8 \%)$ & 0.069 \\
\hline Arthralgia & $28(76 \%)$ & $117(89 \%)$ & 0.046 \\
\hline Arthritis & $9(24 \%)$ & $64(48 \%)$ & 0.009 \\
\hline Hemoglobin, g/dL & $12.5 \pm 0.2$ & $11.4 \pm 0.1$ & $<.001$ \\
\hline Leukocytes, / / L & $7295.1 \pm 523.5$ & $14,708.8 \pm 599.3$ & $<.001$ \\
\hline Neutrophils, / $\mu \mathrm{L}$ & $4714.7 \pm 451.3$ & $12,734.8 \pm 594.8$ & $<.001$ \\
\hline lymphocyte, / $\mu \mathrm{L}$ & $1838.0 \pm 154.6$ & $1100.9 \pm 48.0$ & $<.001$ \\
\hline Platelets, $\times 10^{3} \mu \mathrm{L}$ & $274.0 \pm 16.2$ & $301.1 \pm 11.8$ & 0.26 \\
\hline $\mathrm{ESR}, \mathrm{mm} / \mathrm{h}$ & $40.4 \pm 5.2$ & $64.8 \pm 2.6$ & $<.001$ \\
\hline CRP, mg/dL & $4.3 \pm 1.1$ & $11.6 \pm 0.7$ & $<.001$ \\
\hline $\mathrm{AST}, \mathrm{mg} / \mathrm{dL}$ & $56.5 \pm 17.4$ & $93.3 \pm 17.4$ & 0.065 \\
\hline $\mathrm{ALT}, \mathrm{mg} / \mathrm{dL}$ & $66.7 \pm 21.7$ & $94.2 \pm 21.7$ & 0.28 \\
\hline Ferritin, mg/dL & $721.4 \pm 246.0$ & $6007.6 \pm 887.3$ & 0.002 \\
\hline $\mathrm{LDH}, \mathrm{mg} / \mathrm{dL}$ & $334.0 \pm 52.8$ & $593.5 \pm 103.0$ & 0.21 \\
\hline ANA positivity & $8(29 \%)$ & $21(17 \%)$ & 0.16 \\
\hline RF positivity & $3(9 \%)$ & $10(8 \%)$ & 0.85 \\
\hline Systemic score & $2.6 \pm 0.2$ & $5.0 \pm 0.1$ & $<.0001$ \\
\hline
\end{tabular}

AOSD adult-onset Still's disease, ESR erythrocyte sedimentation rate, CRP C-reactive protein, $A S T$ aspartate transaminase, $A L T$ alanine transaminase, $L D H$ lactate dehydrogenase, $A N A$ antinuclear antibody, $R F$ rheumatoid factor. All values are presented as the number (\%) or the mean $\pm \mathrm{SE}$ except the age as the mean $\pm \mathrm{SD}$. Systemic scores (0-12) were obtained using the method described by Pouchot et al. [7], with 1 point assigned for each of the following manifestations: fever, typical rash, pleuritis, pneumonia, pericarditis, hepatomegaly or abnormal liver function tests, splenomegaly, lymphadenopathy, leukocytosis $\geq 15,000 / \mathrm{mm}^{2}$, sore throat, myalgia, and abdominal pain

and arthritis (48\%), which appeared more frequently in the AOSD group than in non-AOSD patients. The frequencies of other clinical symptoms, such as sore throat and lymphadenopathy, were not significantly different between the two groups. Mean leukocyte and neutrophil counts were significantly higher in AOSD patients $(p<0.001)$, who also had higher ferritin $(p=0.002)$, ESR $(p<0.001)$, and CRP $(p<0.001)$ levels. Patients with AOSD were treated with corticosteroids (95\%), nonsteroidal anti-inflammatory drugs (83\%), methotrexate (52\%), hydroxychloroquine (31\%), IVIG (19\%), a TNF inhibitor (6\%), and IL-6 blockade (3\%). During the follow-up period, 35 patients $(27 \%)$ had disease relapse, 11 patients $(8 \%)$ were treated in the intensive care unit (ICU), and 4 (3\%) died (Table 2). Of the 134 AOSD patients, 17 (13\%) were diagnosed with macrophage activation syndrome (MAS). Three patients died of pneumonia with sepsis during the treatment, and one patient died of fulminant hepatitis related to AOSD.

\section{Concordance between Yamaguchi and ILAR criteria in patients with suspected AOSD}

Concordance between the Yamaguchi and ILAR criteria in patients with suspected AOSD was good $(k=0.7144$, $p<0.001)$ whereas in the diagnosed AOSD group it was fair $(\mathrm{k}=0.3787, p<0.001)$, and lower than that based on a comparison with all suspected AOSD patients (Table 3). Thus, overall, there was substantial concordance between the two sets of criteria. We evaluated the sensitivity and specificity of the ILAR criteria for systemic JIA in patients with suspected AOSD. The 
Table 2 Treatment and prognosis of the patients with adultonset Still's disease (AOSD) during follow-up

\begin{tabular}{llll}
\hline \multicolumn{3}{c}{ AOSD patients $(N=132)$} & AOSD patients \\
Medication & & Prognosis & \\
\hline NSAIDs & $109(83 \%)$ & Relapse & $35(27 \%)$ \\
Corticosteroid & $126(95 \%)$ & MAS & $17(13 \%)$ \\
DMARD & $88(67 \%)$ & ICU & $11(8 \%)$ \\
HCQ & $41(31 \%)$ & Death & $4(3 \%)$ \\
Azathioprine & $18(14 \%)$ & Treatment step & \\
Sulfasalazine & $11(8 \%)$ & 1 & $1(1 \%)$ \\
Leflunomide & $7(5 \%)$ & 2 & $27(20 \%)$ \\
Methotrexate & $69(52 \%)$ & 3 & $70(53 \%)$ \\
IVIG & $25(19 \%)$ & 4 & $34(26 \%)$ \\
TNF inhibitor & $8(6 \%)$ & & \\
IL-6 blockade & $4(3 \%)$ & & \\
\hline AOSD adult-onser & &
\end{tabular}

AOSD adult-onset Still's disease, NSAIDs nonsteroidal anti-inflammatory drugs, DMARD disease modifying antirheumatic drugs, HCQ hydroxychloroquine, IVIG intravenous immunoglobulin, TNF tumor necrosis factor, IL-6 interleukin-6, MAS macrophage activation syndrome, ICU intensive unit care. Values are expressed as $n(\%)$

sensitivity was 0.8864 , with a $95 \%$ confidence interval (CI) of $0.8322-0.9405$, and the specificity was 0.7838 , with a $95 \%$ CI of 0.6511-0.9164 (Table 4).

\section{Correlation between disease activity markers and JIA and Yamaguchi criteria positivity in AOSD patients}

We evaluated correlations between the levels of disease activity markers, such as the systemic score, ESR, and CRP, and the number of positive JIA or Yamaguchi criteria in patients with AOSD. The systemic score $(\rho=0.437, p<0.0001)$, leukocyte count $(\rho=0.312, \quad p=0.0003), \quad$ ESR $(\rho=0.178, \quad p=0.0406)$, CRP level $(\rho=0.216, p=0.0128)$, and LDH level $(\rho=-0.307$, $p=0.0056)$ correlated positively and significantly with the Yamaguchi criteria. The relationships between the

Table 3 Agreement between the Yamaguchi and International League Against Rheumatism (ILAR) criteria in patients with suspected adult-onset Still's disease (AOSD) or with confirmed AOSD

\begin{tabular}{ll}
\hline & Yamaguchi and ILAR criteria \\
\hline All suspected AOSD & \\
Kappa ( $p$-value) & $0.7144(<0.0001)$ \\
95\% confidence interval & $(0.5947,0.8341)$ \\
AOSD-diagnosed patients & \\
Kappa ( $p$-value) & $0.3787(<0.0001)$ \\
95\% confidence interval & $(0.1416,0.6159)$
\end{tabular}

The concordance between the Yamaguchi and ILAR criteria was assessed by the kappa $(\mathrm{k})$ value: a k-value $>0.8$ indicated excellent agreement, $0.61-0.8$ meant good agreement, 0.21-0.4 indicated fair agreement, and $<0.2$ was considered poor agreement
Table 4 Sensitivity and specificity of International League Against Rheumatism (ILAR) and Yamaguchi criteria in suspected adult-onset Still's disease (AOSD) patients

\begin{tabular}{llllllll}
\hline Disease & \multicolumn{2}{l}{ ILAR criteria } & & \multicolumn{2}{l}{ Yamaguchi criteria } & \\
\cline { 2 - 3 } & + & - & & + & - & Total \\
\hline AOSD patients & 117 & 15 & & 119 & 13 & 132 \\
Not AOSD patients & 8 & 29 & & 3 & 34 & 37 \\
Total & 125 & 44 & & 122 & 47 & 169
\end{tabular}

The sensitivity was $88.6 \%$, and the specificity was $78.4 \%$, with the ILAR criteria. The sensitivity was $90.2 \%$, and the specificity was $91.9 \%$, with the Yamaguchi criteria

ILAR criteria for systemic JIA and the systemic score ( $\rho=0.741, p<0.0001)$, CRP level $(\rho=0.237, p=0.0063)$, and albumin $(\rho=-0.281, p=0.0011)$ were also significant and positive (Table 5 ).

\section{Positivity of the ILAR criteria for systemic JIA and the Yamaguchi criteria as a prognostic factor in AOSD patients}

The frequencies of relapse, MAS, care in the ICU, and death were designated as prognostic factors and then the data were analyzed using the logistic regression model with the positivity of the ILAR and Yamaguchi criteria in patients with AOSD. None of the prognostic factors were significantly associated with the Yamaguchi criteria but the relationships of

Table 5 Correlation between disease activity markers and the number of positive International League Against Rheumatism (ILAR) and Yamaguchi criteria in AOSD patients

\begin{tabular}{|c|c|c|c|c|}
\hline & \multicolumn{2}{|c|}{ Yamaguchi criteria } & \multicolumn{2}{|c|}{ ILAR criteria } \\
\hline & $\rho$ & $P$-value & $\rho$ & $P$-value \\
\hline Systemic score & 0.437 & $<0.0001$ & 0.741 & $<0.0001$ \\
\hline Leukocytes & 0.312 & 0.0003 & -0.007 & 0.9369 \\
\hline Hemoglobin & -0.062 & 0.4802 & -0.092 & 0.2953 \\
\hline Platelets & 0.155 & 0.0764 & -0.102 & 0.2461 \\
\hline ESR & 0.178 & 0.0406 & 0.115 & 0.1890 \\
\hline CRP & 0.216 & 0.0128 & 0.237 & 0.0063 \\
\hline Ferritin & 0.169 & 0.0534 & 0.055 & 0.5274 \\
\hline $\mathrm{LDH}$ & -0.307 & 0.0056 & -0.055 & 0.6290 \\
\hline Total bilirubin & -0.150 & 0.0864 & -0.002 & 0.9798 \\
\hline Albumin & -0.156 & 0.0744 & -0.281 & 0.0011 \\
\hline AST & -0.104 & 0.2334 & -0.035 & 0.6891 \\
\hline ALT & -0.032 & 0.7151 & -0.058 & 0.5064 \\
\hline
\end{tabular}

ESR erythrocyte sedimentation rate, $C R P$ C-reactive protein, $L D H$ lactate dehydrogenase, AST aspartate aminotransferase, $A L T$ alanine aminotransferase. Systemic scores (0-12) were obtained using the method described by Pouchot et al. [8], with 1 point assigned for each of the following manifestations: fever, typical rash, pleuritis, pneumonia, pericarditis, hepatomegaly or abnormal liver function tests, splenomegaly, lymphadenopathy, leukocytosis $\geq 15,000 / \mathrm{mm}^{2}$, sore throat, myalgia, and abdominal pain. The Pearson correlation statistic was used to compare the correlation between ESR, CRP, ferritin, and leukocyte counts, as disease activity markers, and the Yamaguchi and ILAR criteria 
relapse (odds ratio $=1.712,95 \% \mathrm{CI}=1.166-2.512$ ), MAS (odds ratio $=1.993,95 \% \mathrm{CI}=1.218-3.263$ ) and ICU care (odds ratio $=2.080,95 \% \mathrm{CI}=1.135-3.811$ ) with the positivity of the ILAR criteria for systemic JIA in patients with AOSD were significant (Table 6).

\section{Discussion}

This is the first study to evaluate the ILAR criteria for systemic JIA in patients with AOSD. The results showed fair concordance between the Yamaguchi and ILAR criteria for systemic JIA in patients with AOSD. However, the positivity of the ILAR criteria may be useful for the identification of AOSD patients at high risk for relapse, association with MAS and ICU care.

Several studies have compared children and adults with Still's disease [18-20], but most have been unable to find specific differences between AOSD and systemic JIA. One study showed that nine patients with systemic JIA met the Yamaguchi criteria [19], while another detected differences with respect to articular involvement between the two groups [18]. Nonetheless, given the large number of similarities between pediatric and adult patients, the diseases can be considered to the same or at least similar. Therefore, we evaluated the sensitivity and specificity of the ILAR criteria for systemic JIA in patients with suspected AOSD. Our results showed concordance between the Yamaguchi and ILAR criteria in these patients. Among the 169 patients with suspected AOSD, Cohen's kappa was 0.7144, indicative of substantial agreement between the two sets of criteria. When only the 132 AOSD patients were considered, Cohen's kappa was lower (0.387), but it still supported agreement between the Yamaguchi and ILAR criteria. There was substantial concordance when the entire patient population with suspected AOSD was included, but only fair concordance when this was limited to the AOSD group. This is probably due to the fact that arthritis was more common in the AOSD group than in the nonAOSD group, but it was still present in less than $50 \%$ of cases. This may reflect the ILAR criteria requiring the presence of arthritis to diagnose a patient with systemic JIA [12]. One study has suggested that the presence of arthritis is a strict criterion, but this could lead to an unacceptable delay in diagnosis [16]. That study also showed that patients with systemic JIA may experience a significant period during which they do not have arthritis, particularly at disease onset [16]. Those authors concluded that the Yamaguchi criteria may be useful for the subset of patients in the pre-arthritic phase of the disease. As part of this process, Dr. Martini proposed a prospective research plan for establishing a new provisional classification system for JIA in the 23rd European Pediatric Rheumatology Congress [15]. In this study, our patients with AOSD had a low frequency of arthritis, which may explain the low sensitivity and specificity of the ILAR criteria in patients with AOSD.

Many recent studies have sought to identify simple markers for evaluating AOSD disease severity, activity, or prognosis. The candidate biomarkers included simple clinical laboratory markers, such as ESR, CRP, ferritin, the neutrophil to lymphocyte ratio, and procalcitonin, as well as several cytokines or chemokines, such as IL-6, IL-8, CXC motif chemokine 13, and IL-18 [5, 21-23]. However, clinical laboratory markers are relatively nonspecific, and cytokine determinations are not clinically feasible. A recent study suggested that Pouchot's systemic score could predict a poor outcome in patients with AOSD. Thus, a score $>7$ and the presence of any complication, such as MAS, kidney failure, or myocarditis at diagnosis, were associated with mortality [24]. Our study evaluated the positivity of the ILAR criteria in patients with AOSD, with respect to preexisting disease activity markers and prognosis. ILAR criteria positivity correlated with the systemic scores and the levels of

Table 6 Positivity of the International League Against Rheumatism (ILAR) criteria and Yamaguchi criteria as a prognostic factor for death or care in the intensive care unit (ICU) in patients with adult-onset Still's disease (AOSD). The data were analyzed using a logistic regression model

\begin{tabular}{|c|c|c|c|c|c|c|}
\hline \multirow{3}{*}{$\begin{array}{l}\text { Outcome } \\
\text { of } \\
\text { interests }\end{array}$} & \multicolumn{6}{|l|}{$\underline{\text { Effect }}$} \\
\hline & \multicolumn{3}{|c|}{ Positive Yamaguchi criteria } & \multicolumn{3}{|l|}{ Positive ILAR criteria } \\
\hline & Odds ratio estimate & \multicolumn{2}{|c|}{$\begin{array}{l}95 \% \text { Wald } \\
\text { confidence interval }\end{array}$} & Odds ratio estimate & \multicolumn{2}{|c|}{$\begin{array}{l}95 \% \text { Wald } \\
\text { confidence limits }\end{array}$} \\
\hline \multicolumn{7}{|l|}{ Prognosis } \\
\hline Relapse & 1.295 & 0.729 & 2.298 & 1.589 & 1.043 & 2.421 \\
\hline MAS & 1.325 & 0.750 & 2.340 & 1.993 & 1.218 & 3.263 \\
\hline $\mathrm{ICU}$ & 1.145 & 0.464 & 2.827 & 2.087 & 1.086 & 4.011 \\
\hline Death & 0.769 & 0.217 & 2.726 & 0.746 & 0.236 & 2.364 \\
\hline
\end{tabular}


CRP and albumin. We were also able to demonstrate an association between positivity for the ILAR criteria and two prognostic factors, relapse and ICU care, in patients with AOSD. These results suggest that the ILAR criteria can aid the evaluation of disease activity and prediction of prognosis of AOSD. The differences between the ILAR and Yamaguchi criteria are the presence of serositis and absence of leukocytosis, sore throat, and elevated liver enzyme concentrations. Our results could be related to the presence of serositis in the ILAR criteria. Some studies suggested that serositis is a poor prognostic factor for early onset JIA or joint damage in systemic JIA $[25,26]$.

There were several important limitations to this study. First, because it was conducted in a single center, the data were limited and may have been subject to selection bias. Second, because we reviewed electronic medical records, some patient data may have been missing and the records of a few patient records were not available. Third, the patients who did not meet the initial screening criterion regarding the diagnostic code for AOSD were not included.

\section{Conclusion}

Despite the lack of sensitivity and specificity of the ILAR criteria for systemic JIA in the classification of AOSD, there was fair concordance between the Yamaguchi and ILAR criteria in AOSD patients. Our results indicate that the ILAR criteria can be used as an additional predictor of relapse, MAS and ICU care in AOSD patients. Further studies of larger populations from several centers are needed to confirm our results regarding the utility of the ILAR criteria in AOSD patients.

\section{Abbreviations}

ALT: Alanine transaminase; ANA: Antinuclear antibody; AOSD: Adult-onset Still's disease; AST: Aspartate transaminase; CBC: Complete blood count; Cl: Confidence interval; CRP: C-reactive protein; DMARD: Disease-modifying antirheumatic drugs; ESR: Erythrocyte sedimentation rate; FUO: Fever of unknown origin; HCQ: Hydroxychloroquine; ICU: Intensive unit care; IL-18: Interleukin 18; ILAR: International League Against Rheumatism; IVIG: Intravenous immunoglobulin; JIA: Juvenile idiopathic arthritis; $\mathrm{k}$ : kappa; LDH: Lactate dehydrogenase; RF: Rheumatoid factor; SD: Standard deviation; SE: Standard error; TNF: Tumor necrosis factor

\section{Acknowledgements}

None

\section{Funding}

This research was supported by a grant of the Korea Health Technology R\&D Project through the Korea Health Industry Development Institute (KHIDI), funded by the Ministry of Health \& Welfare, Republic of Korea (grant number: H16C0992).

\section{Availability of data and materials}

All data generated or analyzed during this study are included in this article.

\section{Authors' contributions}

JWY, EL, J-YS, J-YJ, C-HS and H-AK designed the study. JWY, J-YS and H-AK collected the data. JWY and EL analysed the data under supervision of $\mathrm{H}-\mathrm{AK}$. JWY, EL and H-AK produced the initial draft and all authors contributed to subsequent drafts and approved the final version.

\section{Ethics approval and consent to participate}

This study was approved by the Institutional Review Board of our hospital, and waved to inform the consent by the Institutional Review Board of our hospital because of retrospective study nature.

\section{Consent for publication}

Not applicable

\section{Competing interests}

No author has any potential conflict of interest with respect to the research, authorship or publication of this article.

\section{Publisher's Note}

Springer Nature remains neutral with regard to jurisdictional claims in published maps and institutional affiliations.

\section{Author details}

'Department of Rheumatology, Ajou University School of Medicine, 164 Worldcup-ro, Yeongtong-gu, Suwon 16499, South Korea. ${ }^{2}$ Department of Biomedical Informatics, Ajou University School of Medicine, 164 Worldcup-ro, Yeongtong-gu, Suwon 16499, South Korea.

Received: 9 January 2018 Accepted: 18 January 2018

Published online: 25 January 2018

\section{References}

1. Bywaters EG. Still's disease in the adult. Ann Rheum Dis. 1971;30:121-33.

2. Hedrich $C M$, Günther $C$, Aringer M. Still's disease in children and adults. Z Rheumatol. 2017;76:595-608

3. de Graaff $L C$, ten Broek MR, Schweitzer DH. Is Still's disease still one disease? A case of adult-onset Still's disease showing accumulation in the carotids and the large vessels of the legs on positron emission tomography: CT images. Rheumatol Int. 2012;32:2487-90.

4. Jamilloux Y, Gerfaud-Valentin M, Martinon F, et al. Pathogenesis of adultonset Still's disease: new insights from the juvenile counterpart. Immunol Res. 2015;61:53-62.

5. Inoue N, Shimizu M, Tsunoda S, et al. Cytokine profile in adult-onset Still's disease: comparison with systemic juvenile idiopathic arthritis. Clin Immunol. 2016;169:8-13.

6. Nigrovic PA, Raychaudhuri S, Thompson SD. Review: genetics and the classification of arthritis in adults and children. Arthritis Rheumatol. 2018;70:7-17.

7. Yamaguchi M, Ohta A, Tsunematsu T, et al. Preliminary criteria for classification of adult Still's disease. J Rheumatol. 1992;19:424-30.

8. Pouchot J, Sampalis JS, Beaudet F, et al. Adult Still's disease: manifestations, disease course, and outcome in 62 patients. Medicine (Baltimore). 1991;70:118-36.

9. Nirmala N, Brachat A, Feist E, et al. Gene-expression analysis of adult-onset Still's disease and systemic juvenile idiopathic arthritis is consistent with a continuum of a single disease entity. Pediatr Rheumatol Online J. 2015;13:50.

10. Lebrun $D$, Mestrallet $S$, Dehoux $M$, et al. Validation of the Fautrel classification criteria for adult-onset Still's disease. Semin Arthritis Rheum. 2018:47:578-85

11. Masson C, Le Loet $X$, Liote F, et al. Comparative study of 6 types of criteria in adult Still's disease. J Rheumatol. 1996:23:495-7.

12. Petty RE, Southwood TR, Manners $P$, et al. International league of associations for rheumatology classification of juvenile idiopathic arthritis: second revision, Edmonton, 2001. J Rheumatol. 2004;31:390-2.

13. Fink CW. Proposal for the development of classification criteria for idiopathic arthritides of childhood. J Rheumatol. 1995;22:1566-9.

14. Ravelli A, Varnier GC, Oliveira S, et al. Antinuclear antibody-positive patients should be grouped as a separate category in the classification of juvenile idiopathic arthritis. Arthritis Rheum. 2011;63:267-75.

15. Martini A. It is time to rethink juvenile idiopathic arthritis classification and nomenclature. Ann Rheum Dis. 2012;71:1437-9.

16. Kumar S, Kunhiraman DS, Rajam L. Application of the Yamaguchi criteria for classification of "suspected" systemic juvenile idiopathic arthritis (sJA). Pediatr Rheumatol Online J. 2012:10:40.

17. Landis JR, Koch GG. An application of hierarchical kappa-type statistics in the assessment of majority agreement among multiple observers. Biometrics. 1977;33:363-74.

18. Lin SJ, Chao HC, Yan DC. Different articular outcomes of Still's disease in Chinese children and adults. Clin Rheumatol. 2000;19:127-30. 
19. Luthi F, Zufferey P, Hofer MF, et al. "adolescent-onset Still's disease": characteristics and outcome in comparison with adult-onset Still's disease. Clin Exp Rheumatol. 2002;20:427-30.

20. Uppal SS, Pande IR, Kumar A, et al. Adult onset Still's disease in northern India: comparison with juvenile onset Still's disease. Br J Rheumatol. 1995;34:429-34.

21. Han JH, Suh CH, Jung JY, et al. Association of CXCL10 and CXCL13 levels with disease activity and cutaneous manifestation in active adult-onset Still's disease. Arthritis Res Ther. 2015;17:260.

22. Seo JY, Suh CH, Jung JY, et al. The neutrophil-to-lymphocyte ratio could be a good diagnostic marker and predictor of relapse in patients with adultonset Still's disease: a STROBE-compliant retrospective observational analysis. Medicine (Baltimore). 2017:96:e7546.

23. Chen DY, Lan JL, Lin FJ, et al. Proinflammatory cytokine profiles in sera and pathological tissues of patients with active untreated adult onset Still's disease. J Rheumatol. 2004;31:2189-98.

24. Ruscitti P, Cipriani P, Masedu F, et al. Adult-onset Still's disease: evaluation of prognostic tools and validation of the systemic score by analysis of 100 cases from three centers. BMC Med. 2016;14:194.

25. Russo RA, Katsicas MM. Patients with very early-onset systemic juvenile idiopathic arthritis exhibit more inflammatory features and a worse outcome. J Rheumatol. 2013:40:329-34.

26. Sandborg C, Holmes TH, Lee T, et al. Candidate early predictors for progression to joint damage in systemic juvenile idiopathic arthritis. J Rheumatol. 2006;33:2322-9.

\section{Submit your next manuscript to BioMed Central and we will help you at every step:}

- We accept pre-submission inquiries

- Our selector tool helps you to find the most relevant journal

- We provide round the clock customer support

- Convenient online submission

- Thorough peer review

- Inclusion in PubMed and all major indexing services

- Maximum visibility for your research

Submit your manuscript at www.biomedcentral.com/submit 